\title{
Three km Track Time Trial Performance Changes after HIIT in Competitive Cross-Country Skiers
}

\author{
Anneke Winegarden ${ }^{1}$, Constance Lebrun ${ }^{2}$, Louis Passfield ${ }^{3}$, Patricia Doyle-Baker ${ }^{4}$ \\ ${ }^{1}$ Faculty of Kinesiology, University of Calgary, AB, Canada \\ ${ }^{2}$ Professor, Faculty of Medicine and Dentistry, University of Alberta, Edmonton, Canada \\ ${ }^{3}$ Adjunct Professor, Faculty of Kinesiology, University of Calgary, AB, Canada, Professor, Endurance Research Group, School of Sport \\ and Exercise Sciences, University of Kent, Chatham Maritime, Kent, UK \\ ${ }^{4}$ Professor, Faculty of Kinesiology, University of Calgary, 2500 University Drive, Calgary, AB., T2N 1N4, Canada
}

Corresponding Author: Patricia Doyle-Baker, Dr. PH/PhD, MA, BSc (Hon), E-mail: pdoyleba@ucalgary.ca

\begin{tabular}{l} 
ARTICLE INFO \\
\hline Article history \\
Received: November 03, 2020 \\
Accepted: January 21, 2021 \\
Published: January 31, 2021 \\
Volume: 9 Issue: 1
\end{tabular}

Conflicts of interest: None.

Funding: None.

\begin{abstract}
Background: Limited research surrounding sex differences in fatigue and performance after high intensity interval training (HIIT) exists in the field setting. While lab-based protocols provide a controlled environment, physiological mechanisms of fatigue and performance response in competitive athletes are best measured by time-trial (TT). Few studies, however, have investigated fatigue in TT performance while controlling for the menstrual cycle (MC). Objective: The study investigated the influence of sex and MC phase on $3 \mathrm{~km}$ track TT performance after a HIIT session in Competitive Cross Country (XC) skiers. Methods: A quasi-experimental design was employed and athletes over the age of 16 years $(30 \mathrm{~F}, 9 \mathrm{M})$, were recruited from across Canada and completed 3 days of testing/training: a $3 \mathrm{~km}$ track TT on Day 1 and Day 3; and a HIIT session $(4-8 \mathrm{x}, 800 \mathrm{~m})$ on Day 2. MC phase was verified by ovulation testing and salivary hormone samples; athletes were classified as either "Low Hormone" or "High Hormone" for analysis. Results: An overall improvement in performance from pre- to post-HITT TTs occurred $(p<0.01)$. No significant differences in TT performance after HIIT were observed between sexes $(p=0.16)$ or MC phase $(\mathrm{p}=0.26)$. Conclusion: These results indicate that competitive XC skiers will likely experience an improvement in TT performance after a bout of HIIT. Coaches and athletes should plan their workouts prior to testing accordingly to maximize TT performance. These results also suggest that no special adjustments need to be considered for differences in performance after HIIT between sexes and MC phase, although further studies with a greater sample size and repeated testing are warranted.
\end{abstract}

Key words: High Intensity Interval Training, Sex Differences, Menstrual Cycle Phase, Cross-Country Skiers

\section{INTRODUCTION}

Cross-country (XC) skiing is a popular area for sport science research in Scandinavian countries, but there is a lack of studies on competitive/elite Canadian XC skiers, particularly female athletes. How athletes, such as $\mathrm{XC}$ skiers respond to training and how that may impact their performance during a competition in the outdoors is largely under studied despite the significant evidence available from lab-research on endurance training principles.

Field research unlike the lab environment often involves single-subject research designs to predict performance of an elite athlete. According to Kinugasa (2013), the single-subject approach is effective when there are individual differences in the response to a specific intervention or training. Given the lack of studies completed in the field, more research is needed to elevate the quality of evidence related to field testing for competitive/elite athletes, particularly in the Canadian context.

Training program periodization is an elaborate coordination of all training components (mental skills, skill acquisition, training load, diet, and recovery) to ensure an optimal environment for the competitive athlete (Mujika, Halson, Burke, Balangue \& Farrow, 2018). Traditionally, training load periodization has been a primary focus for coaches and physiologists to maximize training adaptation and allow the athlete to improve the physical attributes that impact performance (Mujika et al., 2018). High Intensity Interval Training (HIIT) is used by endurance athletes to improve performance by targeting physiological adaptations such as increased mitochondrial content, increased capillary density and maximum cardiac output (Blomqvist \& Saltin, 1983; Basset \& Howley, 2000). HIIT has been shown to improve these metabolic and cardiovascular 
performance metrics after 2-8 weeks of 2-3 HIIT sessions per week resulting in performance improvements of 2-4\% in well trained athletes (Daussin, Zoll, Dufour, Ponsot, Lonsdorfer-Wolf, Doutreleau, et al, 2008; Helgerud Hoydal, Wang, Karlsen, Berg, Bjerkass, et al, 2007; Laursen, 2010; Price, Smith, Turner, Waldman and Chander et al., 2020; Talanian, Galloway, Heigenhauser, Bonen \& Spriet, 2007). Endurance training programs typically combine HIIT with low intensity volume training to enhance performance outcomes in sport-specific competition (Hydren \& Cohen, 2015; Hebisz, Hebisx, Borkowski \& Zaton, 2019). HIIT does cause fatigue if appropriate recovery is not prescribed, and chronic training-induced fatigue can lead to overreaching or overtraining which may impact performance outcomes (Kellmann, 2010).

Fatigue can be both perceived and performance based (Enoka \& Duchateau, 2016). The etiology of fatigue can be challenging when studying athletes since many factors simultaneously influence their cognitive and physical performance (Enoka \& Duchateau, 2016). For this reason, in this study fatigue is considered from a whole-body, multiple system view, with decreased time-trial (TT) performance as the key indicator of athlete fatigue.

Time trialling is a special type of performance because mental strength and pacing strategies play an integral role in performance in elite endurance athletes. $3 \mathrm{~km}$ track TTs are used to measure improvements in fitness and performance throughout a skier's season or career (Nordiq Canada, 2018). TTs are known to be reliable and valid performance assessments and are specific to "real-life" races where athletes are required to cover a pre-determined distance at a self-selected pace as fast as they can (Currel \& Jenkendrup, 2008). Performance changes can be measured by repeating a TT before and after a specific intervention. A performance decrement or improvement, post intervention indicates a functional negative or positive effect of an intervention, respectively. Previous studies show performance decrements can detect changes in performance due to muscle fatigue and mental fatigue (Marcora, Bosio \& de Morree, 2008; Marcora, Staiano \& Manning, 2009).

Recent studies demonstrate that men have greater performance fatigability than women in isometric contractions and lower velocity in repeated dynamic tasks, however sex differences in performance fatigability seem to be task dependant (Hunter, 2016). Additionally, males record a longer recovery time after a fatiguing exercise compared to females (Albert, Wrigley, McLean \& Sleivert, 2006). There is limited research, however describing how sex can impact the body's response to a HIIT session and if fatigue manifests differently in men vs women during and after a HIIT session. While men continue to outperform women in shorter duration running events, women have demonstrated they are capable of out-performing men in ultra-distance events, suggesting the possibility that male and female energy systems respond differently (Brown, 2017; Cheuvront, Carter, DeRuisseau \& Moffatt, 2005; Knechtle, Valeri, Nikolaidis, Zingg, Rosemann, \& Rust, 2016). Despite the seeming advantage women have in improved fatigue resistance, female athletes are often prescribed a lower intensity training load in comparison to their male counterparts.

Female-specific research has shown that a woman's metabolism, exercise capacity, and physiological responses to heat can be altered depending on which menstrual cycle (MC) phase they are in (Meendering, Torgrimson, Houghton, Halliwill \& Minson, 2005; Minson, Halliwill, Young \& Joyner, 2000; Oosthuyse, \& Bosch, 2010). Changes in performance across the MC are hypothesized because of the role estrogen and progesterone have on the cardiovascular and respiratory systems as well substrate metabolism and heat regulation (Constantini \& Lebrun, 2005). The MC can be separated into two distinct phases, luteal and follicular, which are divided by ovulation (Oosthuyse \& Bosch, 2010). Estrogen levels rise and fall twice during the MC, first during the mid-follicular phase and then drops dramatically after ovulation. This is followed by a secondary rise in estrogen levels during the mid-luteal phase with a decrease at the end of the MC (see Table 1). The secondary rise in estrogen parallels the rise of progesterone (Reed \& Carr 2018). The luteal phase can be described as the "high hormone phase" whereas the follicular phase can be described as the "low hormone phase" (Striker, Eberhart, Chevailler, Quinn, Bischof, \& Striker, 2006). Between phases estrogen remains high but progesterone drops making for a unique hormone profile around ovulation, which can be studied as its own phase, although is often not considered (Constantini \& Lebrun, 2005). Estrogen plays a role in regulating muscle glycogen storage capacity and free fatty acid availability that can alter exercising metabolism in comparison to when estrogen levels are low (Oosthuyse \& Bosch, 2010). When performance is measured in a TT, increased carbohydrate metabolism in the mid-follicular phase appears to be beneficial for short duration high intensity TTs (Campbell, Angus \& Febbraio, 2001). This suggests the possibility that an increase in estrogen can be beneficial for TT performance (Oosthuyse, Bosch \& Jackson, 2005). Progesterone plays a role in regulating resting heart rate (Sedlak, Shufelt, Iribarren \& Merz, 2012), basal body temperature and ventilation (Charkoudian et al., 1999). Exercise tolerance in hot environments may be reduced when progesterone is high in the luteal phase, although with a more experienced athlete, the impact is likely to be less (Janse de Jonge, Thompson, Chuter, Silk \& Thom, 2012; Tze-Huan et al., 2017).

Therefore, the objective of this study was to determine if sex and MC phase influence performance after a HIIT

Table 1. Hormone phases categorized into low and high hormone adapted from Baird \& Fraser (1974)

\begin{tabular}{lccc}
\hline Hormones & $\begin{array}{c}\text { Early } \\
\text { Follicular } \\
\text { Low hormone }\end{array}$ & Preovulatory & $\begin{array}{c}\text { Mid luteal } \\
\text { High } \\
\text { hormone }\end{array}$ \\
\hline $\begin{array}{l}\text { Progesterone } \\
(\mu \mathrm{g})\end{array}$ & 1 & 4 & 25 \\
Estrone $(\mu \mathrm{g})$ & 50 & 350 & 250 \\
$\begin{array}{l}\text { Estradiol } \\
(\mu \mathrm{g})\end{array}$ & 36 & 380 & 250 \\
\hline
\end{tabular}

Abbreviations: $\mathrm{mg}=$ milligrams; $\mu \mathrm{g}=$ micrograms 
session. Based on the existing literature, we hypothesized that fatigue would impact performance and differences between sexes and menstrual cycle phases would exist.

\section{METHODS}

\section{Participants}

Participants were recruited by communicating with club and provincial coaches across Canada. All XC skiers 16 years of age and older were invited to participate. The inclusion criteria were: 1) 16-35 years of age, 2) training year-round to optimize performance in XC skiing, and 3) competed (or intended to compete) at a national level competition or greater. No specific MC inclusion criteria were set because of the high prevalence of MC irregularity in endurance athletes (De Cree, 1998; Beals \& Manore, 2002). Given that this was a pilot study and effect sizes were not known because of the small athlete population size in this sport, a sample size calculation was not performed. Our recruitment targets were 50 women ( 25 testing in the follicular phase; 25 testing in the luteal phase) and 25 men. These targets were chosen based on expected feasibility and access to the target population. The athletes were fully informed of all experimental procedures before providing written informed consent to participate. The study was performed according to the Declaration of Helsinki. The research was approved by the Conjoint Health Research Ethics Board of the University of Calgary (REB19-0311).

Sixty-three competitive XC skiers from nine different teams agreed to participate. Two participants did not meet the inclusion criteria, 17 participants did not start the testing protocol because of injury/illness or schedule conflicts, and five participants were unable to complete the testing protocol because of injury/illness (see Table 2).

\section{Study Design}

A quasi-experimental design was employed to investigate sex and menstrual cycle differences in TT performance after a HIIT workout. The protocol design mimicked a typical 3-4 day summer training camp where running takes on large focus of training. Athletes completed the protocol on three consecutive days in the general preparation phase of their training program, and on a week that was deemed appropriate by their coach (typically following a rest week). Instructions were given to not do any structured workouts on the testing days as well as the day immediately prior to testing and athletes completed the testing protocol with their teammates at their typical training time (varied based on team). Testing sessions took place typically on the local $400 \mathrm{~m}$ track, however the quality of the track varied based on location ranging from competition grade tracks in larger cities to dirt tracks in smaller towns. Within each testing block, testing times remained consistent within the constraints of each specific location and considerations were made to perform testing sessions in similar weather conditions. Athletes were asked to verbally report questionnaires scores indicating their self-reported fatigue (i.e., "How fatigued are you right now") (Rating of Fatigue Questionnaire, Micklewright, St Clair Gibson, Gladwell \& Salman, 2017) and affect (i.e., "How are you feeling right now?") (Feeling Scale, Hardy \& Rejeski, 1989) before, after and 30mins post each session. Maximum and average heart rate data were also collected during each session.

The testing and protocol sequence (see Table 3) involved the athletes being given on Day 2, a lap time that was 5\% faster than Day 1 and they were instructed to run $800 \mathrm{~m}$ reps until they were no longer able to hold that lap time. If an athlete's pace dropped 5\% below the prescribed pace they were instructed to end their workout. The workout structure was intended to accommodate a range in training status. The athletes completed a minimum of four repeats and $77 \%$ completed all eight repetitions.

\section{Determination of Menstrual Cycle Phase}

At least one-month prior to the athlete's testing session the $\mathrm{MC}$ was tracked by two methods. The first involved counting the days between the start of two cycles to determine cycle length. The second method involved ovulation testing performed by the athlete and started on day 10 of their cycle and continued until a positive test result was obtained or until 15 test strips had been used (Clearblue ovulation testing). If an athlete knew that they typically had a cycle length $>30$ days, they were given 20 test strips. Salivary estrogen and progesterone samples were collected on Day 2 of the testing protocol and were used to verify menstrual status (Labrix salivary hormone testing). Hormone kits were obtained from, and analyzed by, Labrix by Doctors Data (Clackamas, OR, USA). If a positive ovulation test occurred and the participant was in the follicular or luteal phase during their testing session, they were classified as "Low Hormone" or "High Hormone", respectively. If the athlete did not obtain a positive ovulation test and had a low salivary estrogen to progesterone ratio ( $<200$ units), they were classified as "Low Hormone". All female athletes were analyzed together and were divided into three groups: low-hormone, high-hormone, and oral contraceptives (active phase and intra-uterine devices (IUD)) for the purpose of analyzing the influence of MC phase.

Table 2. Mean and SD $( \pm)$ self-reported participant characteristics $(\mathrm{n}=39)$

\begin{tabular}{lccccccc}
\hline Sex & $\begin{array}{c}\text { Age } \\
\mathbf{( y r s )}\end{array}$ & $\begin{array}{c}\text { Height } \\
\mathbf{( m )}\end{array}$ & $\begin{array}{c}\text { Weight } \\
(\mathbf{k g})\end{array}$ & $\begin{array}{c}\text { BMI } \\
(\mathbf{k g} / \mathbf{m} 2)\end{array}$ & $\begin{array}{c}\text { Running } \\
(\mathbf{m i n s} / \mathbf{w k})\end{array}$ & $\begin{array}{c}\text { Training } \\
(\mathbf{h r s} / \mathbf{w k})\end{array}$ & CPL \\
\hline $\mathrm{M}(9)$ & $17.7 \pm 2.2$ & $1.8 \pm 0.1$ & $71.4 \pm 7.5$ & $20.9 \pm 1.3$ & $160.5 \pm 100.0$ & $4.0 \pm 6.9$ & $75.7 \pm 6.4$ \\
$\mathrm{~F}(30)$ & $17.9 \pm 2.6$ & $1.7 \pm 0.1$ & $59.9 \pm 6.4$ & $16.9 \pm 8.6$ & $169.5 \pm 76.5$ & $9.1 \pm 3.9$ & $76.3 \pm 4.4$ \\
\hline
\end{tabular}

$\mathrm{BMI}=$ body mass index $\mathrm{CPL}=$ Canadian Points List (Canadian XC ski racing performance indicator, a higher CPL indicates a higher level of performance) 


\section{Outcome Measures}

The $3 \mathrm{~km}$ track TT has been identified by Nordiq Canada (Nordiq Canada, 2018) as a method to test and monitor an athlete's improvement over a season and throughout their career. Performance in the $3 \mathrm{~km}$ track TTs on Day 1 and Day 3 was used to calculate performance change.

$$
\text { Performance Change }=\frac{(\mathrm{TT} 1-\mathrm{TT} 2)}{\mathrm{TT} 1} * 100
$$

A negative performance change is referred to as a "Performance Decrement" and a positive performance change is called "Performance Improvement". Perceived fatigue (Rating of Fatigue Questionnaire; Micklewright et al., 2017) scores (scale of $1-10$, where 10 is the highest level of fatigue) were collected at the beginning and end of each session.

\section{Statistical Analysis}

Data analysis was conducted using the statistical software SPSS v.26 (SPSS, IBM Corp., NY, USA). Data are expressed as mean and standard deviation $(\mathrm{SD}, \pm)$. A two-tailed, one sample t-test was conducted to determine the influence of HIIT on performance change. Two-tailed, unpaired t-tests were conducted to determine differences in mean response between male and female athletes. An ANOVA was used to compare performance change between male, female low-hormone, female high-hormone and female oral-contraceptives. A significance value of $p \leq 0.05$ was set. The magnitude of the mean differences between sex and menstrual status were expressed as effect sizes (ES). The classification of the ES were based on guidelines by Batterham and Hopkins (2006): trivial $(<0.2)$, small $(>0.2-0.6)$, moderate $(>0.6-1.2)$, large $(>1.2-2.0)$, and very large $(>2.0)$.

\section{RESULTS}

The average MC length was 28 days and $33 \%$ of the athletes (10/30) had ovulatory cycles while the remaining 67\% were non-ovulatory (20/30). Salivary hormone samples identified $7 \%$ of the athletes with a balanced hormone profile $(n=2 / 30)$ while $93 \%$ of the women $(n=28 / 30)$ with a low progesterone to estrogen ratio.

Performance improved, on average in $\mathrm{XC}$ skiers after a HIIT session $(\mathrm{PC} \neq 0, \mathrm{p}=0.01)$. Twelve $(11 \mathrm{~F} ; 1 \mathrm{M})$ athletes had a performance decrement whereas 27 (19F; 8M) had a performance improvement. The group mean performance change (see Figure 1 ) was $1.07 \pm 2.58 \%$ with the mean higher in males than females respectively $(2.19 \pm 3.11 \% ; 0.74$ $\pm 2.36 \%)$. Despite men $(8 / 9 ; 88 \%)$ having a $1.45 \%$ greater performance improvement in comparison to women $(27 / 39$; $69 \%$ ), performance change was not significantly different between sexes $(p=0.14)$. The ES between sexes was small but nearing a medium effect size (0.058).

Performance change in female-low hormone, female-high hormone and female-oral contraceptives was 1.11 $\pm 2.00 \%, 0.67 \pm 3.18 \%$, and $-0.81 \pm 1.87 \%$, respectively (see Figure 2). While there appears to be some differences
Table 3. Testing and Protocol Sequence

\begin{tabular}{lll}
\hline Day 1 & Day 2 & Day $\mathbf{3}$ \\
\hline Testing: & Training: & Testing: \\
3000m & Critical Speed & $3000 \mathrm{~m}$ Track TT \\
Track TT & Workout (track) & \\
& $4-8 \times 800 \mathrm{~m}$ with 2 min rest & \\
& $(800 \mathrm{~m}$ lap times $5 \%$ faster & \\
& than Day 1) & \\
\hline TT $=$ Time Trial
\end{tabular}

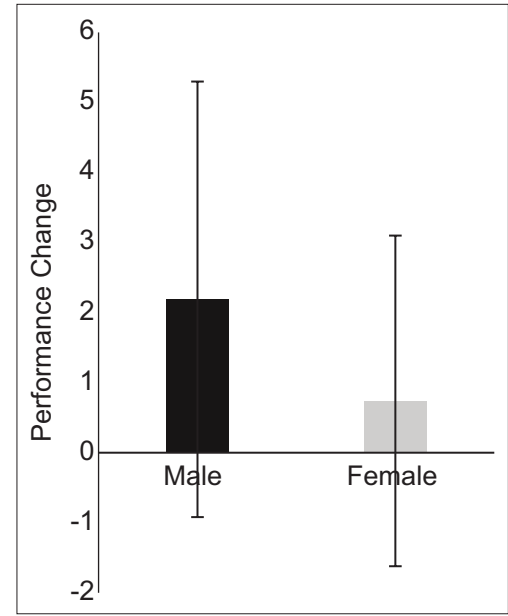

Figure 1. Mean performance change (with SD bars) in males $(n=9)$ and females $(n=30)$



Figure 2. Mean performance change (with SD bars) in male $(\mathrm{n}=9)$, female-low hormone ( $\mathrm{LH} ; \mathrm{n}=18)$, female-high hormone $(\mathrm{HH} ; \mathrm{n}=8)$, and female-oral contraceptive $(\mathrm{OC} ; \mathrm{n}=4)$

between these groups, these results were not statistically significant $(p=0.27)$. The ES between menstrual status was 0.105 which is regarded as a medium effect size. The trend shows a reduced increase in performance in the high hormone group in comparison to the low hormone group, and a performance decrement was observed on average for females taking oral contraceptives.

There was a significant time effect for self-reported rating of fatigue $(R O Q)$ for all athletes $(p<0.0001)$. ROFQ significantly increased from PreTT1 (3.44 \pm 1.37$)$ to PreTT2 (4.74 $\pm 1.56)(\mathrm{p}=0.001)$. ROFQ also significantly increased from 
Pre-HIIT $(3.76 \pm 1.56)$ to PostHIIT $(8.90 \pm 1.31)(\mathrm{p}<0.0001)$ and remained significantly different from PreHIIT at $30 \mathrm{mins}$ PostHIIT $(6.25 \pm 1.75)(\mathrm{p}<0.001)$. A significant reduction in ROF-Q was reported from PostHIIT to 30mins PostHIIT $(\mathrm{p}<0.0001)$. Further, athletes reported a significant reduction in ROF-Q from 30mins PostHIIT to PreTT2 ( $\mathrm{p}=0.001)$. Self-reported fatigue was not influenced by sex $(\mathrm{p}=0.295)$ or menstrual status $(p=0.285)$ and there was no significant interaction between reported fatigue scores and $\operatorname{sex}(\mathrm{p}=0.246)$ or menstrual status $(\mathrm{p}=0.405)$ (see Tables 4 and 5).

There was a significant time effect for self-reported affect (Feeling Scale (FS)) for all athletes $(p=0.001)$ and they reported significant reductions in FS from PreTT1 (1.69 \pm $1.56)$ to PreTT2 $(0.79 \pm 2.04)(\mathrm{p}=0.02)$. While no significant difference in FS was reported from PreHIIT $(1.82 \pm 1.45)$ to PostHIIT $(0.18 \pm 2.44)(p=0.07)$, there was a significant increase in FS from PostHIIT to 30mins PostHIIT (1.08 \pm $2.17)(p=0.04)$. FS was not influenced by sex $(p=0.997)$ or menstrual status $(p=0.238)$ and there was no significant interaction between reported fatigue scores and sex $(\mathrm{p}=0.664)$ or menstrual status $(\mathrm{p}=0.291)$ (see Tables 4 and 5).

\section{DISCUSSION}

The purpose of this study was to investigate whether sex and $\mathrm{MC}$ phase influenced fatigue and training response after a HIIT session in competitive XC skiers. The main finding was that instead of athletes having a performance decrement after a HIIT session, performance, on average, significantly improved.

Table 4. Self-reported rating of fatigue (ROF-Q) and affect (Feeling Scale) grouped by sex

\begin{tabular}{|c|c|c|c|c|c|c|}
\hline Outcome Measure & $\frac{\text { All }}{\text { Mean } \pm \text { SD }}$ & $\begin{array}{c}\text { Male (9) } \\
\text { Mean } \pm \text { SD }\end{array}$ & $\begin{array}{c}\text { Female (30) } \\
\text { Mean } \pm \text { SD }\end{array}$ & $\begin{array}{c}\text { Time Effect } \\
\text { F (df) }[P]\end{array}$ & $\begin{array}{c}\text { Group Effect } \\
\text { F (df) }[\mathrm{P}]\end{array}$ & $\begin{array}{c}\text { Interaction } \\
\text { F (df) }[\mathrm{P}]\end{array}$ \\
\hline \multicolumn{7}{|l|}{ ROF-Q } \\
\hline PreTT1 & $3.44 \pm 1.37$ & $4.00 \pm 1.12$ & $3.10 \pm 1.19$ & \multirow{5}{*}{$\begin{array}{c}66.846(4,34) \\
{[p<0.0001]}\end{array}$} & \multirow{3}{*}{$\begin{array}{l}1.130 \\
(1,37)\end{array}$} & \multirow{3}{*}{$\begin{array}{l}1.428 \\
(4,34)\end{array}$} \\
\hline PreTT2 & $4.74 \pm 1.56$ & $4.78 \pm 1.64$ & $3.31 \pm 1.22$ & & & \\
\hline PreHIIT & $3.76 \pm 1.56$ & $3.56 \pm 1.13$ & $3.80 \pm 1.35$ & & & \\
\hline PostHIIT & $8.90 \pm 1.31$ & $9.00 \pm 1.32$ & $8.93 \pm 1.11$ & & \multirow[t]{2}{*}[\mathrm{p}=0.295]{} & \multirow[t]{2}{*}[p=0.246]{} \\
\hline 30 minsPostHIIT & $6.25 \pm 1.75$ & $7.00 \pm 0.87$ & $6.27 \pm 1.66$ & & & \\
\hline \multicolumn{7}{|l|}{ Feeling Scale } \\
\hline PreTT1 & $1.69 \pm 1.56$ & $1.67 \pm 1.58$ & $1.70 \pm 1.58$ & \multirow{5}{*}{$\begin{array}{c}7.469(4,148) \\
{[p<0.0001]}\end{array}$} & \multirow{4}{*}{$\begin{array}{c}0.000 \\
(1, \mathrm{~s} 37)\end{array}$} & \multirow{4}{*}{$\begin{array}{c}0.599 \\
(4,148)\end{array}$} \\
\hline PreTT2 & $0.79 \pm 2.04$ & $0.78 \pm 2.49$ & $0.80 \pm 1.94$ & & & \\
\hline PreHIIT & $1.82 \pm 1.45$ & $2.00 \pm 1.73$ & $1.77 \pm 1.38$ & & & \\
\hline PostHIIT & $0.18 \pm 2.44$ & $-0.33 \pm 2.29$ & $0.33 \pm 2.50$ & & & \\
\hline 30 minsPostHIIT & $1.08 \pm 2.17$ & $1.44 \pm 2.30$ & $0.97 \pm 2.16$ & & {$[\mathrm{p}=0.997]$} & {$[\mathrm{p}=0.664]$} \\
\hline
\end{tabular}

PreTT1 = measures taken prior to TT on Day 1; PreTT2 = measures taken prior to the TT on Day 3; PreHIT = measures taken prior to the HIIT session on Day 2; PostHIIT = measures taken immediately after the HIIT session on Day 2; 30minsPostHIIT $=$ measures taken 30mins after the HIIT session on Day 2

Table 5. Self-reported rating of fatigue (ROF-Q) and affect (Feeling Scale) grouped by sex and menstrual status

\begin{tabular}{|c|c|c|c|c|c|c|c|}
\hline Outcome Measure & $\frac{\text { Male (9) }}{\text { Mean } \pm \text { SD }}$ & $\begin{array}{c}\text { LH (18) } \\
\text { Mean } \pm \text { SD }\end{array}$ & $\begin{array}{c}\text { HH (8) } \\
\text { Mean } \pm \text { SD }\end{array}$ & $\frac{\mathrm{OC}(4) \_}{\operatorname{Mean} \pm \text { SD }}$ & $\begin{array}{l}\text { Time Effect } \\
\text { F (df) }[\mathrm{P}]\end{array}$ & $\begin{array}{l}\text { Group Effect } \\
\text { F (df) }[\mathrm{P}]\end{array}$ & $\begin{array}{c}\text { Interaction } \\
\text { F (df) [P] }\end{array}$ \\
\hline \multicolumn{8}{|l|}{ ROF-Q } \\
\hline PreTT1 & $4.00 \pm 1.12$ & $3.00 \pm 1.19$ & $3.00 \pm 1.18$ & $3.75 \pm 0.56$ & \multirow{3}{*}{$\begin{array}{l}99.631 \\
(4,140)\end{array}$} & \multirow{3}{*}{$\begin{array}{l}1.315 \\
(3,35)\end{array}$} & \multirow{3}{*}{$\begin{array}{c}1.052 \\
(12,140)\end{array}$} \\
\hline PreTT2 & $4.78 \pm 1.64$ & $4.56 \pm 1.40$ & $5.38 \pm 1.39$ & $4.25 \pm 3.00$ & & & \\
\hline PreHIIT & $3.56 \pm 1.13$ & $3.72 \pm 1.36$ & $4.00 \pm 1.33$ & $3.75 \pm 1.34$ & & & \\
\hline PostHIIT & $9.00 \pm 1.32$ & $8.83 \pm 1.14$ & $9.50 \pm 1.13$ & $8.25 \pm 1.14$ & \multirow[t]{2}{*}[\mathrm{p}<0.0001]{} & \multirow[t]{2}{*}[\mathrm{p}=0.285]{} & \multirow[t]{2}{*}[\mathrm{p}=0.405]{} \\
\hline 30 minsPostHIIT & $7.00 \pm 0.87$ & $6.39 \pm 1.48$ & $6.39 \pm 1.50$ & $5.00 \pm 0.00$ & & & \\
\hline \multicolumn{8}{|l|}{ Feeling Scale } \\
\hline PreTT1 & $1.67 \pm 1.58$ & $1.56 \pm 1.54$ & $1.75 \pm 1.91$ & $2.25 \pm 1.26$ & \multirow{3}{*}{$\begin{array}{c}4.995 \\
(4,140)\end{array}$} & \multirow{3}{*}{$\begin{array}{l}1.477 \\
(3,35)\end{array}$} & \multirow{3}{*}{$\begin{array}{c}1.197 \\
(12,140)\end{array}$} \\
\hline PreTT2 & $0.78 \pm 2.49$ & $1.00 \pm 1.88$ & $-0.13 \pm 2.23$ & $1.75 \pm 0.96$ & & & \\
\hline PreHIIT & $2.00 \pm 1.73$ & $1.83 \pm 1.58$ & $1.50 \pm 1.31$ & $2.00 \pm 0.00$ & & & \\
\hline PostHIIT & $-0.33 \pm 2.29$ & $0.22 \pm 2.29$ & $-0.63 \pm 2.93$ & $2.75 \pm 0.50$ & {$[\mathrm{p}=0.001]$} & {$[\mathrm{p}=0.238]$} & {$[\mathrm{p}=0.291]$} \\
\hline 30 minsPostHIIT & $1.44 \pm 2.30$ & $0.83 \pm 1.95$ & $0.13 \pm 2.42$ & $3.25 \pm 0.96$ & & & \\
\hline
\end{tabular}

PreTT1 = measures taken prior to TT on Day 1; PreTT2 = measures taken prior to the TT on Day 3; PreHIIT = measures taken prior to the HIIT session on Day 2; PostHIIT = measures taken immediately after the HIIT session on Day 2; 30minsPostHIIT $=$ measures taken 30mins after the HIIT session on Day 2 
Our expectation was that the intervention would result in both performance fatigue (changes in contractile function and muscle activation) and perceived fatigue (changes in homeostasis and psychological state), therefore reducing the athlete's ability to perform the TT after a HIIT workout (Enoka \& Duchateau, 2016). Although athletes reported a significant increase in ROF-Q immediately after the HIIT workout, self-reported fatigue had significantly decreased by the following morning. Despite reporting significantly greater ROF-Q and significantly lower FS prior to the TT on Day 3 in comparison to prior to the TT on Day1, athletes significantly improved their TT performance. This finding does not align with measures of muscular fatigue where maximal power output or time exhaustion are reduced in an isolated muscle group after an acute bout of fatiguing exercise (Enoka, \& Duchateau 2011) Rietjes and colleagues (2005) found also that acute fatigue in athletes does not decrease TT performance in the short-term. This suggest that the factors affecting peripheral fatigue are robust and may require a longer period of over-reaching to be impacted. While some aspects of fatigue are well known, there are still gaps in our knowledge on how fatigue translates to human performance and how percieved fatigue can be trained with exercise intervention (Enoka \& Duchateau, 2016).

While most physiological adaptations to HIIT such as increased capillary density and increased cardiac output, require a time frame greater than three days to manifest, adaptations in skeletal muscle mitochondrial content can be triggered with as little as one session of HIIT (MacInnis \& Gibala, 2017; Gibala McGee, Garnham, Howlett, Snow, \& Hargreaves, 2009). Most endurance athletes will train primarily in low to moderate intensities in the general preparation phase, therefore it is possible that the HIIT session created enough stimulus to lead to improvements in performance (Mujika et al., 2018). An additional challenge in field research, is that we cannot isolate physiological and psychological improvements (Noakes, 2000). An athlete's conscious effort and motivation to sustain a given exercise intensity can be significant enough to lead to an increase in performance. Due to the "against the clock" nature of TTs, the athlete's knowledge of their previous time may have motivated them to attain a new personal best. Completing a vigorous intensity exercise routine can increase pain tolerance during exercise, therefore it is possible that an acute adaptation in pain tolerance could have contributed to the increase in performance (Jones, Booth, Taylor \& Barry, 2014). It is also possible that this pilot protocol was so demanding the athletes increased their recovery methods during the testing sessions more than they would have previously. Many athletes anecdotally reported the HIIT session was the hardest workout they had done all season, and they needed an extra nap in the day or went to bed early because they were feeling tired. The recovery status of the athletes is supported by the significant reductions in self-reported fatigue once $30 \mathrm{mins}$ of active recovery is completed after the HIIT session, and further reductions in self-reported fatigue between or after approximately $24 \mathrm{hrs}$ of recovery (prior to TT2). If an athlete improved their recovery methods and was able to recover adequately from the HIIT session, it may result in increased capacity to perform (Bishops, Jones \& Woods, 2008).

Based on the lack of statistical significance, the impact of sex and MC phase on performance after a HIIT session is unclear and speculative at best given the limitations of field based testing. Although men appeared to have a greater improvement in performance and women on OC had a greater performance decrement after HIIT, individual variation in response may actually play a larger role than sex or MC phase. Previous findings, however on sex differences in performance fatigability suggest that women are more fatigue resistant then men in isometric, low intensity muscle contractions, but the difference between is reduced during dynamic tasks (Hunter, 2016). A running TT is a series of dynamic, low intensity muscular contractions, leading us to believe that the mechanism responsible for sex differences in muscular fatigability may not influence training-induced fatigue after HIIT. Unpublished data by Cobbold (2018) showed that after 6-weeks of HIIT training, men had an increase in $\mathrm{VO} 2_{\text {max }}$ by $11 \%$ whereas women did not significantly improve. It may be that men are more susceptible to improved performance after HIIT because of higher testosterone levels in comparison to women. Testosterone has been shown to be significantly elevated after HIIT session in young male athletes (Kilian, Engel, Wahl, Achtzehn, Sperlich, \& Mester, 2016) and Remes and colleagues (1979) documented greater increases in $\mathrm{VO}_{\text {max }}$ after training in men with higher testosterone levels. A running TT is a series of dynamic, low intensity muscular contractions, that may have the potential to demonstrate sex differences in muscular fatigability with a repeated measures design. Sex differences in adaptation to training needs to be further developed to improve our understanding of health and performance benefits of HIIT following both single and multiple HIIT training sessions in endurance athletes such as XC skiers.

The lack of difference between high hormone and low hormone indicates that recovery and fatigue, as well as adaptation from a HIIT session may not be influenced by MC phase. These findings seem to support Hunter's (2016) statement that MC difference in fatigability is far less significant than sex differences, however we recognize that our study design may limit any substantive outcome. Although others have hypothesized that HIIT may be better tolerated in the follicular phase because of increased carbohydrate metabolism and increased relative testosterone (Sims \& Yeager, 2016), the present study is unable to support this because of the small time window that athletes were observed. As well the significance of these findings on the influence of the MC phase is limited because of the high prevalence of anovulatory cycles progesterone insufficiency reported in the salivary samples. It's likely that the majority of athletes would be classified as "oligomenorrheic" with one or more missed cycles throughout the duration of tracking. Some level of menstrual irregularity based on the "unbalanced" estrogen to progesterone ratio existed in all athletes with only two exceptions. The performance decrement in athletes taking OC $(n=4)$, while not significant, has been identified in a training study and a systematic review and meta-analysis. 
Schaumberg and colleagues (2017) found that adaptation of $\mathrm{VO} 2_{\text {max }}$ from sprint-interval training was dampened in women taking OC. Kristy-Ellis and colleagues also found that OC use resulted in slightly inferior exercise performance on average when compared to naturally menstruating women (2020)

A limitation of the study was that repeated measurements were not completed in the luteal phase and the follicular phase (Julian et al., 2017). While repeated measurements could have added to our understanding of how the MC impacts fatigue and training response after HIIT, it was logistically too challenging for athletes to commit to that many testing sessions. Additionally, because of the nature of individual sports training, we were not able to standardize the training and lifestyle of the athletes prior to their testing sessions. While we requested that athletes do the testing following a week of lower training load to ensure fatigue levels were not too great, some athletes reported higher levels of fatigue coming into testing on Day 1. Competitive XC skiers should be familiar with the $3 \mathrm{~km}$ track testing since it is recognized as a standardized test by Nordiq Canada, however some athletes had no experience running it while others had completed several $3 \mathrm{~km}$ tests previously. Therefore, a learning effect cannot be dismissed and may have contributed to athlete's results. Despite some differences in athlete's training history and experience, this sample was likely representative of competitive Canadian XC skier population.

Future studies should consider including more than one sport (i.e., cycling, running, triathlon, $\mathrm{XC}$ skiing) to see if there is a trend that remains true amongst a variety of endurance sports. Based on this study's standard error, a sample of 46 male and 46 female participants would be required to be recruited to detect significant sex differences at a level of $p=0.05$. While finding a significant difference between sex may be possible with more participants, our sample standard error indicates 168 participants are needed for High Hormone and Low Hormone groups to find a significant difference $(p=0.05)$, which may be unrealistic for future studies. Additionally, future research should include a longer period of exhaustive training (greater than 2 weeks), within the "training camp" model as this may be the most appropriate approach to reduce extraneous factors that impact performance.

\section{CONCLUSION}

The results of this field study are influenced by a potential learning effect. This limitation must be considered before stating that competitive XC skiers will likely experience an improvement in TT performance after a bout of HIIT. The trend was towards performance improvement, on average, however we are unable to comment adequately on fatigue. We know that performance change varies between individuals, and it is possible that male athletes may have a greater response to HIIT than women athletes. In this study performance was not influenced by the $\mathrm{MC}$, with the exception of a reduction in performance after HITT in a few athletes taking OC, which may have played a role (Kristy-Ellis et al., 2020).
Coaches may want to consider a training block that allows for recovery after a HIIT session, to maximize testing performance and athletes may want to familiarize themselves with the protocol so that they maximize the potential adaptation to HIIT.

\section{ACKNOWLEDGMENTS}

This study was undertaken in the University of Calgary (Alberta, Canada). Testing sessions were conducted at local track venues in Ottawa, ON., Montreal, QC., Revelstoke, BC., and Calgary, AB. The authors thank the following for their assistance and contribution to this research project: Victor Wiltmann, Maurice Samm, Camille Chesky, Kevin Sheilds, Sylvie Girard, Ron Howden, Rick Dickey, Chris Jeffries, Matt Smider, and Eric Groeneveld. As well as XC ski coaches from Ontario, Quebec, Alberta, and British Columbia who encouraged their athletes to participate and helped with local logistics. Dr. Tak Fung, statistician at the University of Calgary assisted with statistical analysis and interpretation and all the athletes who participated in the study.

\section{AUTHORS' CONTRIBUTIONS}

AW participated in the design of the study, contributed to data collection, data analysis interpretation of results and writing; PKDB participated in the design of the study, contributed to the analysis, interpretation of results and writing; LP participated in the design of the study and contributed to the interpretation of the results; CL participated in the design of the study.

\section{REFERENCES}

Albert, W.J., Wrigley, A.T., McLean, R.B. \& Sleivert, G.G. (2006). Sex differences in rate of fatigue development and recovery. Dynamic Medicine,5(2), 1-10. DOI: 10.1186/1476-5918-5-2

Batterham, A.M. \& Hopkins, W.G. (2006). Making meaningful inferences about magnitudes. International Journal of Sports Physiology and Performance 2006;1:(Suppl. 1):50-57. DOI: 10.1123/ijspp.1.1.50

Baird, D.T. \& Fraser, I.S. (1974, Jun.). Blood production and ovarian secretion rates of estradiol-17 beta and estrone in women throughout the menstrual cycle. The Journal of Clinical Endocrinology and Metabolism, 38(6), 100917. DOI: 10.1210/jcem-38-6-1009. PMID: 4598662.

Beals, K.A. \& Manore, M.M. (2002). Disorders of the female athlete tried among collegiate athletes. International Journal of Sport Nutrition and Exercise Metabolism, 12(3), 281-293. DOI: 10.1123/ijsnem.12.3.281

Bishop, P.A., Jones, E. \& Woods, A.K. (2008). Recovery from training: A brief review. Journal of Strength and Conditioning Research, 22(3), 1015-1024. DOI: 10.1519/JSC.0b013e31816eb518

Blomqvist, C.G. \& Saltin, B. (1983). Cardiovascular adaptations to physical training. Annual Review of Physiology, 45, 169-189. DOI: 10.1146/annurev. ph.45.030183.001125 
Brown, M. (April 11, 2017). The longer the race, the stronger we get. Retrieved from https://www.outsideonline. com/2169856/longer-race-stronger-we-get

Campbell, S.E., Angus, D.J. \& Febbraio, M.A. (2001). Glucose kinetics and exercise performance during phases of the menstrual cycle: effect of glucose ingestion. American Journal of Physiology, 281, E817-825. DOI: 10.1152/ajpendo.2001.281.4.E817

Charkoudian, N., Stephens, D.P., Pirkle, K.C., Kosiba, W.A. \& Johnson, J.M. (1999). Influence of female reproductive hormones on local thermal control of skin blood flow. Journal of Applied Physiology, 87(5), 1719-1723. DOI: $10.1152 /$ jappl.1999.87.5.1719

Cheuvront, A.N., Carter III, R., DeRuisseau, K.C. \& Moffatt, R.J. (2005). Running performance differences between men and women: An update. Sports Medicine, 35(12), 1017-1024. DOI: 10.2165/00007256-200535120-00002

Cobbold, C. (2018). Battle of the sexes: Which is better for you, high- or low-intensity exercise? Journal of Sport and Health Sciences, 7(4), 429-432. DOI: 10.1016/j.jshs.2018.05.004

Constantini, N.W. \& Lebrun, C.M. (2005). The Menstrual Cycle and Sport Performance. Clinical Sports Medicine, e51-e82. DOI: 10.1016/j.csm.2005.01.003

Currell, K. \& Jeukendrup, A.E. (2008). Validity, Reliability and Sensitivity of Measures of Sporting Performance. Sports Medicine, 28(4), 297-316. DOI: 10.2165/00007256-200838040-00003

Daussin, F.N., Zoll, J., Dufour, S.P., Ponsot, E., LonsdorferWolf, E., Doutreleau, S., Mettauer, B., Piquard, F., Geny, B. \& Richard, R. (2008). Effect of interval versus continuous training on cardiorespiratory and mitochondrial functions: relationship to aerobic performance improvements in sedentary subjects. American Journal of PhysiologyRegulatory, Integrative and Comparative Physiology, 295, R264-R272. DOI: 10.1152/ajpregu.00875.2007

De Cree, C. (1998). Sex steroid metabolism and menstrual irregularities in the exercising female: A review. Sports Medicine, 25(6), 369-406. DOI: 10.2165/00007256-199825060-00003

Elliott-Sale, K.J., McNulty, K.L., Ansdell, P. et al. (2020). The Effects of Oral Contraceptives on Exercise Performance in Women: A Systematic Review and Meta-analysis. Sports Medicine, 50, 1785-1812. https:// doi.org/10.1007/s40279-020-01317-5

Enoka, B.M. \& Duchateau, J. (2016). Translating Fatigue to Human Performance. Medicine \& Science in Sports \& Exercise, 48(11), 2228-2238. DOI: 10.1249/ MSS.0000000000000929

Gibala, M.J., McGee, S.L., Garnham, A.P., Howlett, K.F., Snow, R.J. \& Hargreaves, M. (2009). Brief intense interval exercise activates AMPK and p38 MAPK signaling and increases the expression of PGC-1 in human skeletal muscle. Journal of Applied Physiology, 106, 929-934. DOI: 10.1152/japplphysiol.90880.2008

Hardy, C.J. \& Rejeski, W.J. (1989). Not what, but how one feels: The measurement of affect during exercise. Journal of Sport \& Exercise Psychology, 11(3), 304-317.
Hebisz, R., Hebisz, P., Borkowski, J. \& Zaton, M. (2019). Effects of concomitant high-intensity interval training and sprint interval training on exercise capacity and response to exercise- induced muscle damage in mountain bike cyclists with different training backgrounds. Isokinetics \& Exercise Science, 27 (5), 1-9. DOI: 10.3233/IES-183170

Helgerud, J., Hoydal, K., Wang, E., Karlsen, T., Berg, P.L., Bjerkass, M., Simonsen, T., Helgesen, C., Hjorth, N., Bach, R. \& Hoff, J. (2007). Aerobic high-intensity intervals improve VO2max more than moderate training. Medicine \& Science in Sports Exercise, 39(4), 665-671. DOI: $10.1249 / \mathrm{mss} .0 \mathrm{~b} 013 \mathrm{e} 3180304570$

Hunter, S.K. (2016). The relevance of sex differences in performance fatigability. Medicine \& Science in Sports \& Exercise, 48(11), 2247-2256. 10.1249/ MSS.0000000000000928

Hydren, J.R. \& Cohen, B.S. (2015). Current scientific evidence for a polarized cardiovascular endurance training model. Journal of Strength and Conditioning Research, 29(12), 3523-3530. DOI: 10.1519/ JSC.0000000000001197

Janse de Jonge, X.,A.,K., Thompson, M.W., Chuter, V.H., Silk, L.N. \& Thom, J.M. (2012). Exercise performance over the menstrual cycle in temperate and hot, humid conditions. Medicine \& Science in Sports \& Exercise, 44(11), 2190-2198. DOI: 10.1249/MSS.0b013e3182656f13

Jones, M.D., Booth, J., Taylor, J.L. \& Barry, B.K. (2014). Aerobic training increases pain tolerance in healthy individuals. Medicine \& Science in Sports \& Exercise, 46(8), 1640-1647. DOI: 10.1249/MSS.0000000000000273

Julian, R., Hecksteden, A., Fullagar, H.H.K. \& Meyer, T. (2017). The effects of menstrual cycle phase on physical performance in female soccer players. PloS ONE, 12(3): e0173951. DOI: 10.1371/journal.pone.0173951

Kellman, M. (2010). Preventing overtraining in athletes in high-intensity sports and stress/recovery monitoring. Scandinavian Journal of Medicine \& Science in Sports, 20 (2), 95-102. DOI: 10.1111/j.1600-0838.2010.01192.x

Kilian, Y., Engel, F., Wahl, P., Achtzehn, S., Sperlich, B. \& Mester, J. (2016). Markers of biological stress in response to a single session of high-intensity interval training and high-volume training in young athletes. European Journal of Applied Physiology, 116, 21772186. DOI: 10.1007/s00421-016-3467-y

Kinugasa, Y. (2013). The Application of Single-Case Research Designs to Study Elite Athletes' Conditioning: An Update. Journal of Applied Sport Psychology, 25(1), 157-166. https://doi.org/10.1080/10413200.2012.709578

Knechtle, B., Valeri, F., Nikolaidis, P.T., Zingg, M.A., Rosemann, T. \& Rust, C.A. (2016). Do women reduce the gap to men in ultra-marathon running? SpringerPlus, 5, 672-687. DOI: 10.1186/s40064-016-2326-y

Laursen, P.B. (2010). Training for intense exercise performance: high intensity or high volume training? Scandinavian Journal of Medicine \& Science in Sports, 20 (Suppl. 2): 1-10. DOI: 10.1111/j.1600-0838.2010.01184.x 
MacInnis, M.J. \& Gibala, M.J. (2017). Physiological adaptations to interval training and the role of exercise intensity. The Journal of Physiology, 595(9), 2915-2930. DOI: 10.1113/JP273196

Marcora, S. M., Bosio, A. \& de Morree, H. M. (2008). Locomotor muscle fatigue increases cardiorespiratory responses and reduces performance during intense cycling exercise independently from metabolic stress. American Journal of Physiology-Regulatory, Integrative and Comparative Physiology, 294 (3), R874. DOI: 10.1152/ajpregu.00678.2007

Marcora, S.M., Staiano, W. \& Manning, V. (2009). Mental fatigue impairs physical performance in humans. Journal of Applied Physiology, 106, 857-864. DOI: 10.1152/ japplphysiol.91324.2008

Mattu, A.T., Iannetta, D., MacInnis, M.J., Doyle-Baker, P.K. \& Murias, J.M. (2019). Menstrual and oral contraceptives cycle phases do not affect submaximal and maximal exercise responses. Scandinavian Journal of Medicine \& Science in Sports, 30(3), 472-484. DOI: 10.1111/sms. 13590

Meendering, J.R., Torgrimson, B.N., Houghton, B.L., Halliwill, J.R. \& Minson, C.T. (2005). Menstrual Cycle and sex affect hemodynamic responses $\mathrm{t}$ combined orthostatic and heat stress. American Journal of Sports Physiology. Heart \& Circulatory Physiology, 289(2), H631-H642. DOI: 10.1152/ajpheart.00029.2005

Micklewright, D., St Clair Gibson, A., Gladwell, V. \& Salman, A. (2017). Development and validity of the rating-of-fatigue scale. Sports Medicine, 47, 2375-2393. DOI: $10.1007 / \mathrm{s} 40279-017-0711-5$

Minson, C.T., Halliwill, J.R., Young, T.M., \& Joyner, M.J. (2000). Influence of the menstrual cycle on sympathetic activity, baroreflex sensitivity, and vascular transduction in young women. Circulation, 101, 862-868. DOI: 10.1161/01.cir.101.8.862

Mujika, I., Halson, S., Burke., L.M., Balangue, G., \& Farrow, D. (2018). An integrated, multifactorial approach to periodization for optimal performance in individual and team sports. International Journal of Sports Physiology, 13(5), 538-561. DOI: 10.1123/ijspp.2018-0093

Noakes, T.D. (2000). Physiological models to understand exercise fatigue and the adaptations that predict or enhance athletic performance. Scandinavian Journal of Medicine \& Science in Sports, 10, 123-145. DOI: 10.1034/j.1600-0838.2000.010003123.x

Nordiq Canada. (2018). Run jump pull dryland testing. Retrieved from https:/www.cccski.com/Programs/ Athlete-Development/Runjumppull-dryland-test.aspx

Oosthuyse, T. \& Bosch, A.N. (2010). The effect of the menstrual cycle on exercise metabolism: Implications for exercise performance in eumenorrheic women. Sports Medicine, 40(3), 207-27. DOI: $10.2165 / 11317090-000000000-00000$

Oosthuyse, T., Bosch, A.N. \& Jackson, S. (2005). Cycling time trial performance during different phases of the menstrual cycle. European Journal of Applied Physiology, 94(3), 268-76. DOI: 10.1007/s00421-005-1324-5
Price, F.G., Smith, J.W., Turner, A.J., Krings, B.M., Waldman, H.S., Chander, H.,... \& McAllister, M.J. (2020). High-Intensity Interval Training in MiddleDistance NCAA Division I 800/1500m Collegiate Athletes. International Journal of Kinesiology and Sports Science, 8(3), 28-35.

Reed, B.G. \& Carr, B.R. (2018). The Normal Menstrual Cycle and the Control of Ovulation. [Updated 2018 Aug 5]. In: Feingold KR, Anawalt B, Boyce A, et al., editors. Endotext [Internet]. South Dartmouth (MA): MDText.com, Inc.; 2000-. Available from: https://www. ncbi.nlm.nih.gov/books/NBK279054/

Remes, K., Kuoppasalmi, K. \& Adlercreutz, H. (1979). Effect of long-term physical training on plasma testosterone, androstenedione, luteinizing hormone and sex-hormone-binding globulin capacity. Scandinavian Journal of Clinical Laboratory Investigations, 39(8), 743-749. DOI: 10.1080/00365517909108166

Rietjens, G.J.M., Kulper, H., Adams, J.J., Sarks, W.H.M., van Breda, E., van Hamont, D. \& Keizer, H.A. (2005). Physiological, biochemical and psychological markers of strenuous training-induced fatigue. International Journal of Sports Medicine, 26, 16-26. DOI: $10.1055 / \mathrm{s}-2004-817914$

Schaumberg, M.A., Jenkins, D.G., Janse de Jonge, X.A., Emmerton, L.M. \& Skinner, T.L. (2017). Oral contraceptive use dampens physiological adaptations to sprint interval training. Medicine \& Science in Sports \& Exercise, 49(4), 717-727. DOI: 10.1249/MSS.0000000000001171

Sedlak, T., Shufelt, C., Iribarren, C., \& Merz, C.N. (2012). Sex hormones and the QT interval: A review. Journal of Women's Health, 21(9), 933-941. DOI: 10.1089/ jwh.2011.3444

Sims, S. \& Yeager, S. (2016). Roar: How to match your food and fitness to your unique female physiology for optimum performance, great health, and a strong, lean body for life. Rodale Inc. USA. pp. 8, 19-20.

Striker, R., Eberhart, R., Chevailler, MC., Quinn, F.A., Bischof, P. \& Striker, R. (2006). Establishment of detailed reference values for luteinizing hormone, follicle stimulating hormone, estradiol, and progesterone during different phases of the menstrual cycle on the Abbott ARCHITECT ${ }^{\circledR}$ analyzer. Clinical Chemistry and Laboratory Medicine (CCLM), 44(7), 883-887. DOI: 10.1515/CCLM.2006.160

Talanian, J.L., Galloway, S.D.R., Heigenhauser, G.J.F., Bonen, A. \& Spriet, L.L. (2007). Two weeks of high-intensity aerobic interval training increases the capacity for fat oxidation during exercise in women. Journal of Applied Physiology, (Suppl. 4), 1439-1447. DOI: 10.1152/japplphysiol.01098.2006

Tze-Huan, L. Stannard, S.R., Perry, B., Schlader, Z., Cotter, J.D. \& Mundel, T. (2017). Influence of menstrual phase and arid vs humid heat stress on autonomic and behavioural thermoregulation during exercise in trained but unacclimated women. Journal of Physiology, 595(9), 2823-2837. DOI: 10.1113/JP273176 